\title{
Drug resistance in tuberculosis
}

\author{
D.A. Mitchison
}

ABSTRACT: A drug-resistant strain of Mycobacterium tuberculosis is defined as one differing from the tight distribution of wild strains that have not come into contact with the drug concerned.

Sensitivity tests are performed by the absolute concentration method, the resistance ratio method or the proportion method. The hypothesis underlying the proportion method is that there are appreciable differences in inoculum size so that there should be an association between the proportion on drug-free medium and the proportion on drug-containing medium. This hypothesis was not supported by a study on ethionamide-resistant strains. It indicated that variation in the proportion on drug-free medium was due to clumping of the bacilli in the inoculum rather than to differences in the number of bacilli.

Hence, the use of the proportion method introduces errors in susceptibility testing. While the method can produce reliable results, it is more time consuming than a minimal inhibitory concentration determination, and should not be adopted as a standard method.

KEYWORDS: Drug resistance, prognosis, tuberculosis

\section{WHAT IS MEANT BY DRUG RESISTANCE?}

Unlike many bacterial species, there is usually remarkably little variation in the susceptibility of different strains of Mycobacterium tuberculosis to the drugs used in first-line treatment [1, 2]. For this reason, it is possible to consider a distribution of the minimal inhibitory concentration (MIC) of "wild" strains that have never come into contact with the drug and, from this distribution, fix a cut-off MIC that distinguishes between those "sensitive" strains that fall within the distribution and those "resistant" strains that have higher MICs, so that they have a chance of, say, $<1 \%$ of being within the distribution. Since wild strains are so uniform in sensitivity, the resistant strains could only have arisen during the treatment of a patient and are, therefore, capable of growth in patients given the drug concerned in monotherapy. The general adoption of this definition avoided some of the pitfalls in thinking, such as the possibility that there was a difference between "laboratory" and "clinical" resistance. While it is still the best way of defining resistance for rarely used drugs, such as those in use for reserve drug treatment, the occurrence of appreciable proportions of strains with primary resistance amongst pre-treatment strains made it necessary to adopt a discriminant statistical technique, which measures the optimal MIC for discriminating between two groups of strains, one that is probably sensitive (PS) and obtained pre-treatment, and the other that is predominantly resistant (PR) and likely to contain a fairly high proportion of resistant strains. These are the two fundamental ways of defining drug resistance [3].

\section{SENSITIVITY TEST METHODS}

During the 1960s, there was much discussion about the methods used in drug sensitivity tests (DSTs) because of discrepant results between laboratories. The World Health Organization (WHO) called two meetings with international participants to discuss the techniques and their uses $[4,5]$. These meetings reported accounts of three different methods for performing DSTs: the absolute concentration method, the resistance ratio method and the proportion method. The rationale behind the proportion method, described by the Paris Pasteur Institute [5], was as follows. A standard inoculum was prepared from strains on Lowenstein-Jensen medium by

Previous articles in this series: No. 1: Cardona P-J, Ruiz-Manzano J. On the nature of Mycobacterium tuberculosis-latent bacilli. Eur Respir J 2004; 24: 1044-1051. No. 2: Rieder H. Annual risk of infection with Mycobacterium tuberculosis. Eur Respir J 2005; 25: 181-185.

AFFILIATIONS

Medical Microbiology, St George's Hospital Medical School, London, UK.

CORRESPONDENCE

D.A. Mitchison

Medical Microbiology

St George's Hospital Medical School Cranmer Terrace

London SW17 ORE

UK

Fax: 442086720234

E-mail: dmitchis@sghms.ac.uk

Received:

June 232004

Accepted:

July 132004 
shaking the growth with glass beads and then adjusting the opacity to a standard. When counts of colony-forming units (cfu) were obtained by the usual serial dilution method on these suspensions, there was substantial variation from strain to strain. This was considered to be the cause of variation in DST results, since some tests would have been inoculated with a large inoculum that would grow on higher drug concentrations, while others would have received a small inoculum able to grow only on lower concentrations.

The hypothesis of a strong association between the proportion on drug-free medium and growth on drug-containing medium was questioned in the original report, and has only been critically examined once in a comparison of methods for tests against ethionamide [6]. Accurate ethionamide tests have always been difficult to obtain, partly because the change in MICs associated with resistance is small and partly because the drug is thermolabile. Hence, the distributions of PS and PR strains are not well separated (fig. 1). The inoculum in this study was prepared by taking growth assessed from its appearance on a loop as weighing $4 \mathrm{mg}$ and shaking this with glass beads to give the standard inoculum. Tests were then set up with serial dilutions of the inoculum suspension, such that MICs, resistance ratios and proportions could be calculated. According to the Pasteur Institute hypothesis [5], there should have been a strong association between the cfu count on a drug-free medium and the MIC on $10 \mu \mathrm{g} \cdot \mathrm{mL}^{-1}$ ethionamide, while there should be no association between the drug-free cfu count and the proportion. This is the opposite of the actual findings (fig. 2). The findings are, however, consistent with the hypothesis that variation in cfu counts on a drug-free medium is due almost entirely to the amount of clumping of the bacilli in suspensions. Looking at a diagrammatic representation of test results on a well-dispersed and a clumped suspension (fig. 3), it can be observed that the two-fold difference between the counts on a drug-free medium is not accompanied by any change in the number of resistant organisms growing on a drug-containing medium, indicating no association between drug-free count and MIC, while a two-fold decrease in

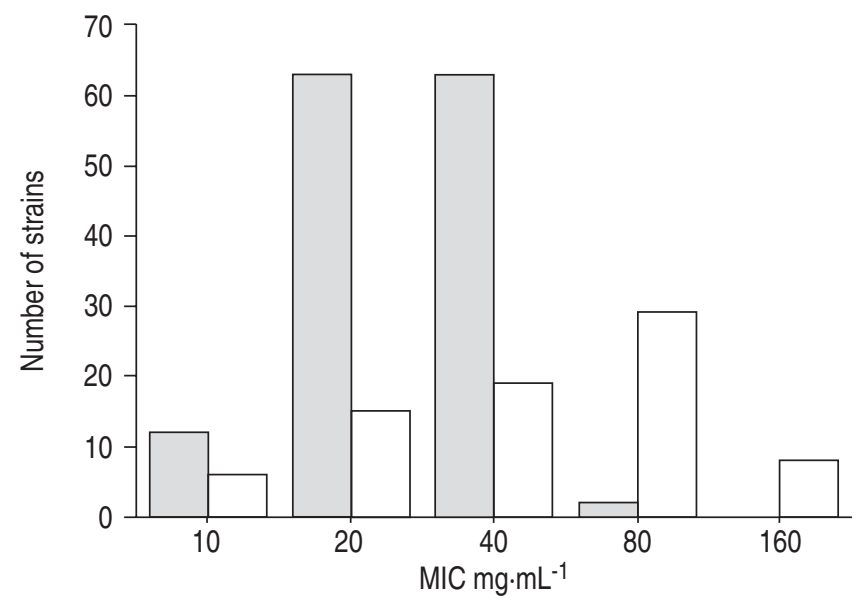

FIGURE 1. The minimal inhibitory concentrations (MICs) of ethionamide of probably sensitive $(\square)$ strains, obtained pre-treatment from patients, and strains that are probably resistant $(\square)$, obtained after treatment of patients with ethionamide. Data taken from [6]
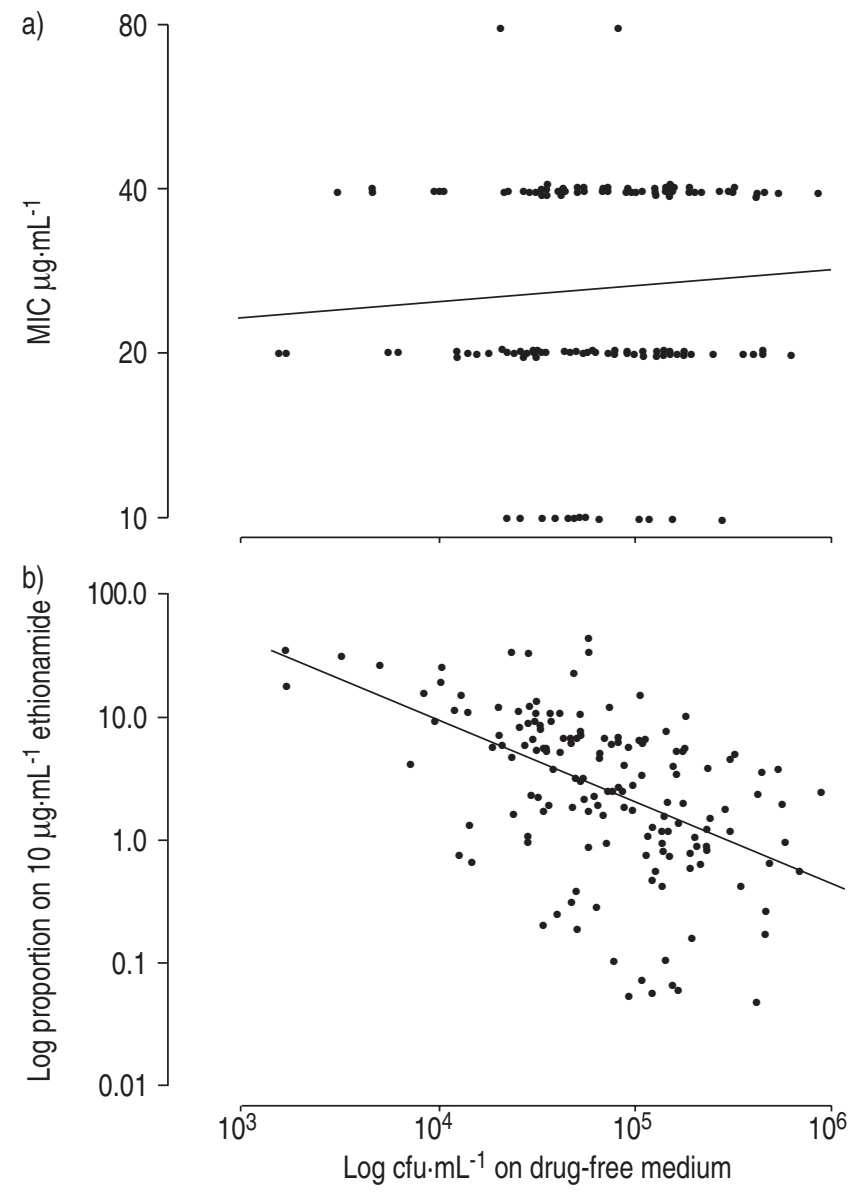

FIGURE 2. Associations between colony-forming unit (cfu) count on drug-free medium and a) minimal inhibitory concentration (MIC) of ethionamide or b) proportion growing on 10 slopes containing $10 \mu \mathrm{g} \cdot \mathrm{mL}^{-1}$ ethionamide amongst pretreatment strains. Association in a) is nonsignificant ( $p>0.05)$, while association in b) is highly significant $(p<0.001)$. Data taken from [6].

drug-free count between the clumped and dispersed inoculum is accompanied by a two-fold increase in the proportion on a drug-containing medium. These findings clearly indicate that the hypothesis on which the proportion tests were designed is wrong and that the use of proportions actually introduces errors. When the methods were compared, the combined proportion definition of resistance of either $\geqslant 50 \%$ growing on $10 \mu \mathrm{g} \cdot \mathrm{mL}^{-1}$ or $\geqslant 10 \%$ on $20 \mu \mathrm{g} \cdot \mathrm{mL}^{-1}$ indicated resistance in $43 \%$ of the $77 \mathrm{PR}$ cultures, while the standard inoculum MIC indicated $48 \%$. By both methods, only one out of 140 PS cultures was designated as resistant.

Thus, the proportion definition was, if anything, slightly less efficient than the MIC method. Resistance ratios were much less efficient because they are calculated from two titrations, one on the test strain and the other on strain H37Rv. The result is subject to the errors in both titrations.

\section{CONCLUSIONS ON ALTERNATIVE SENSITIVITY TEST METHODS}

In practice, the proportion method seems to yield reasonably accurate results in experienced hands. However, there are two 


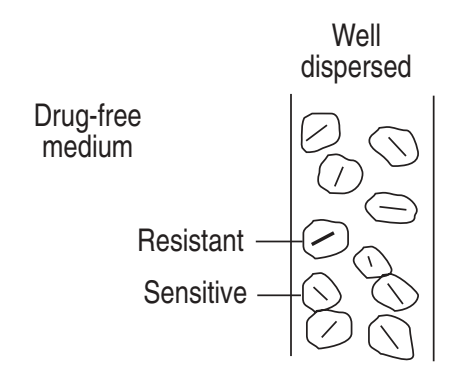

10 colonies

Proportion:

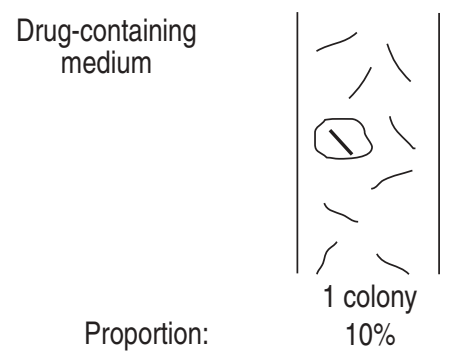

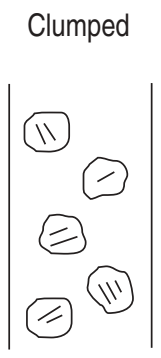

5 colonies

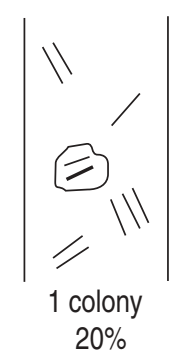

FIGURE 3. Diagrammatic effect of clumping of inoculum suspension on the calculation of the proportion. Each of the four slopes is inoculated with 10 bacilli, which give rise to 10 colonies from the well-dispersed inoculum suspension but to five colonies from the clumped suspension. However, both of the suspensions yield one colony on drug-containing slopes. The calculated proportion is then $10 \%$ with the well-dispersed suspension, but is wrongly estimated as $20 \%$ with the clumped suspension, even though the true proportion is $10 \%$. The minimal inhibitory concentration remains the same for both suspensions, since one colony grows on drug-containing medium. Modified from [7].

reasons why it should never have been adopted as a standard procedure, as follows. 1) The time spent on a proportion test is far greater than on an MIC test, since serial dilutions have to be set up and colonies counted. 2) The criteria for resistance are set from the work (never detailed) carried out many years ago at the Pasteur Institute in Paris, and there is no check on whether the definitions are valid for the laboratory, often in a developing country, where the test is used. While tests for many drugs are fairly stable, others, particularly for streptomycin and other aminoglycosides, are influenced by factors such as access of oxygen during culture and the method of inspissation. What this means is that the tests add greatly to the backlog of work often found in developing countries' laboratories and yield results of uncertain reliability. The current author believes that it is time to consider whether proportion tests should continue to be considered as standard tests by the WHO and whether they might be replaced by simpler tests that are at least as accurate.

\section{RECOMMENDATIONS FOR DRUG SENSITIVITY TESTS}

It is recommended that laboratories using Lowenstein-Jensen medium for tests adopt the following procedures standardised by the British Medical Research Council on the grounds of efficiency and safety [8]: 1) use screw-capped $28 \mathrm{~mL}$ bottles for culture and sensitivity tests, mainly because they bounce when dropped on the floor, whereas glass test tubes break, producing a major hazard; and 2) use a well-designed inspissator capable of providing uniform heating of the bottles (or tubes) for $60 \mathrm{~min}$ at $85^{\circ} \mathrm{C}$. Hot-air ovens, which distribute heat unevenly through the metal rods supporting medium tubes and not through the air, should be avoided. MIC DSTs should then be set up with an inoculum that yields $\sim 1 \times 10^{5}$ cfu added with a loop to each slope, and the tests should be read after incubation for 4 weeks. Given these conditions, reliable results can be obtained with tests that use a single drug-containing slope for each slope, though it is advisable to set up the test on two strains obtained at much the same time or in duplicate. The critical concentrations for screening tests are $0.2 \mu \mathrm{g} \cdot \mathrm{mL}^{-1}$ isoniazid, $32 \mu \mathrm{g} \cdot \mathrm{mL}^{-1}$ rifampicin, $16 \mu \mathrm{g} \cdot \mathrm{mL}^{-1}$ streptomycin and $2.8 \mu \mathrm{g} \cdot \mathrm{mL}^{-1}$ ethambutol. If $>20$ colonies grow on these concentrations, the strain is considered resistant. However, it is wise to check on the critical concentration for streptomycin by titrating a small sample of pre-treatment strains, remembering that dihydrostreptomycin is more stable, but is also more difficult to obtain. Rapid tests that titrate the MIC in liquid or on solid media may well be as accurate as such a standard test. Indeed, they appear to be as good as proportion tests, even when carried out against reserve drugs of low activity [9].

THE LIMITATION OF THE 1\% PROPORTION DEFINITION Other types of test purport to use proportion methods [10], but the practice of defining resistance as the ability of, for example, $1 \%$ of the strain to grow on a particular drug concentration is really equivalent to an MIC test with a small inoculum. There is no evidence that a decrease in inoculum size increases the ability of tests to discriminate between sensitive and resistant strains, apart from pyrazinamide tests, which behave quite differently to DSTs on other drugs [11]. The criticism that has been raised of the proportion method does not apply to the BacTec method, since it is much less likely to be influenced by clumping, but, again, there seems little justification in a method that dilutes the inoculum 100-fold in the test. If the aim of a proportion test is to find out what proportion of the organisms is resistant and what proportion is still sensitive in heterogeneous strains, it would be better to set up a range of dilutions only on slopes containing the critical drug concentration rather than to calculate an inaccurate proportion from dilutions on drug-free medium.

\section{PROGNOSTIC VALUE OF DRUG SENSITIVITY TESTS}

The prognostic value of drug sensitivity test results depends on the bactericidal action of the drug in question during therapy with the drug regimen used for the patient. In the 8month regimens that use thiacetazone or ethambutol with isoniazid in the continuation phase, isoniazid is the major sterilising drug during the continuation phase. Consequently, the response in patients with initial resistance is substantially inferior to the response in patients with sensitive organisms (table 1). In contrast, when the continuation phase is with rifampicin and isoniazid, rifampicin does all of the actual killing during this phase. As a result, initial resistance to isoniazid does not affect response (table 2). In either case, isoniazid reduces by 10 -fold the count of viable bacilli in the sputum during the first 2 days [12], but has no further action during the initial phase when killing is due to rifampicin and pyrazinamide [13]. The initial kill during the first 2 days is insufficient to affect the ultimate response to treatment. There is no information about the prognostic effect of resistance to 


\begin{tabular}{|c|c|c|c|c|}
\hline \multirow{2}{*}{$\begin{array}{l}\text { TABLE } 1 \\
\text { Regimen }\end{array}$} & \multicolumn{4}{|c|}{$\begin{array}{l}\text { Relapses after treatment with a continuation phase } \\
\text { of thiacetazone or ethambutol with isoniazid in } \\
\text { patients with pre-treatment strains sensitive or } \\
\text { resistant to isoniazid }\end{array}$} \\
\hline & $\begin{array}{l}\text { Clinical } \\
\text { trials }\end{array}$ & $\begin{array}{l}\text { Initial susceptibility } \\
\text { to isonazid }\end{array}$ & $\begin{array}{l}\text { Patients } \\
\text { assessed }\end{array}$ & Relapse \\
\hline \multirow[t]{2}{*}{ SHRZ/TH } & 8 & Sensitive & 928 & $71(7.7)$ \\
\hline & & Resistant & 67 & 21 (31.3) \\
\hline \multirow[t]{2}{*}{ EHRZ/EH } & 1 & Sensitive & 420 & $41(9.8)$ \\
\hline & & Resistant & 35 & $11(31.4)$ \\
\hline $\begin{array}{l}\text { Data are pr } \\
\text { isoniazid; } F \\
\text { taken from }\end{array}$ & $\begin{array}{l}\text { ented as } \\
\text { ifampicir } \\
3,14]\end{array}$ & $\begin{array}{l}\text { and } n(\%), \text { unless oth } \\
\text { : pyrazinamide; } \mathrm{T} \text { : }\end{array}$ & $\begin{array}{l}\text { stated. S: s } \\
\text { zone; E: et }\end{array}$ & $\begin{array}{l}\text { mycin; H: } \\
\text { utol. Data }\end{array}$ \\
\hline
\end{tabular}

\begin{tabular}{|c|c|c|c|c|}
\hline TABLE 2 & \multicolumn{4}{|c|}{$\begin{array}{l}\text { Relapses after treatment with a continuation phase } \\
\text { of rifampicin and isoniazid in patients with } \\
\text { pre-treatment strains sensitive or resistant to } \\
\text { isoniazid }\end{array}$} \\
\hline Regimen & $\begin{array}{c}\text { Clinical } \\
\text { trials }\end{array}$ & $\begin{array}{c}\text { Initial susceptibility } \\
\text { to isonazid }\end{array}$ & $\begin{array}{c}\text { Patients } \\
\text { assessed }\end{array}$ & Relapse \\
\hline \multirow[t]{2}{*}{ SHRZ/RH } & 6 & Sensitive & 1225 & $64(5.2)$ \\
\hline & & Resistant & 61 & $5(8.2)$ \\
\hline \multirow[t]{2}{*}{ EHRZ/RH } & 1 & Sensitive & 190 & $7(3.7)$ \\
\hline & & Resistant & 23 & $1(4.0)$ \\
\hline
\end{tabular}

pyrazinamide in standard regimens, because drug sensitivity tests to pyrazinamide are rarely performed in clinical trials. Resistance to rifampicin only, a rare occurrence, still leaves isoniazid available and the response is often good. When there is resistance to both rifampicin and isoniazid (multiple drug resistance disease), the response is substantially inferior, though some response may occur perhaps because pyrazinamide and ethambutol are active during the initial phase. There is no information on the prognostic effect of ethambutol resistance. Since ethambutol may well interfere with the sterilising activity of other drugs in the regimen $[12,13]$, it is possible that resistance to it might slightly improve response.

\section{REFERENCES}

1 Mitchison DA. Problems of drug resistance. Brit Med Bull 1954; 10: 115-124.

2 Selkon JB, Mitchison DA. A concept of drug resistance. Transactions 17th Conference. Chemotherapy of tuberculosis. Washington, Veterans Admin; pp. 258.

3 Mitchison DA. What is drug resistance? Tubercle 1969; 50: 44-47.

4 Canetti G, Froman S, Grosset J, et al. Mycobacteria: laboratory methods for testing drug sensitivity and resistance. Bull World Health Organ 1963; 29: 565-578.

5 Canetti G, Fox W, Khomenko A, et al. Advances in techniques of resting mycobacterial drug sensitivity, and the use of sensitivity tests in tuberculosis control programmes. Bull World Health Organ 1969; 41: 21-43.

6 Lefford MJ, Mitchison DA. Comparison of methods for testing the sensitivity of Mycobacterium tuberculosis to ethionamide. Tubercle 1966; 47: 250-262.

7 Mitchison DA. Sensitivity testing. In: Heaf F, Rusby L, eds. Recent advances in respiratory tuberculosis. 6th Edn. London, Churchill, 1968; pp. 160-182.

8 Mitchison DA. Bacteriology of tuberculosis. Trop Doct 1974; 4: 147-153.

9 Martin A, Camacho M, Portaels F, Palomino JC. Resazurin microtiter assay plate testing of Mycobacterium tuberculosis susceptibilities to second-line drugs: rapid, simple, and inexpensive method. Antimicrob Agents Chemother 2003; 47: 3616-3619.

10 Heifets LB. Drug susceptibility in the chemotherapy of mycobacterial infections. Boca Raton, CRC Press Inc., 1991.

11 Zhang Y, Mitchison DA. The curious characteristics of pyrazinamide: a review. Int J Tuberc Lung Dis 2003; 7: 6-21.

12 Jindani A, Doré CJ, Mitchison DA. The bactericidal and sterilising activities of antituberculosis drugs during the first 14 days. Am J Respir Crit Care Med 2003; 167: 1348-1354.

13 Mitchison DA. Role of individual drugs in the chemotherapy of tuberculosis. Int J Tuberc Lung Dis 2000; 4: 796-806.

14 Jindani A, Nunn AJ, Enarson DA. Two 8-month regimens of chemotherapy for the treatment of newly diagnosed pulmonary tuberculosis: international multicentre randomised trial. Lancet 2004; 364: 1244-1251. 